\title{
The Experimental Research and Analysis of Sugar Mud by Filter Pressing
}

\author{
Lin-feng Zhang ${ }^{a}$,Yan-hui Chen ${ }^{b}$, Guo-jin Xie ${ }^{c}$, Xin-jie Zhang ${ }^{d}$ \\ School of Mechanical Engineering,Guangxi University of Science and Technology,Liuzhou \\ 545006, China

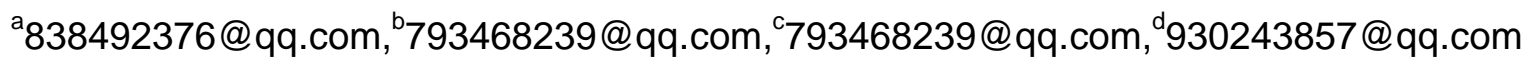

\begin{abstract}
Keywords: filter pressing with variable pressure,constant pressure,moisture,compression ratio Abstract:The experimental research has been carried out about the constant and variable pressure of sugar mud by filter pressing in this paper in order to meet the needs of production, and analyze the changed relationship between the filtrated volume and time under the constant pressure, Under the consideration of filter clothes with various mesh leads to the different results of experiment,taking the experiment of filter pressing under the variable pressure with the appropriate filter cloth to understand the character on sediment, such as moisture,by analyzing the data of experiment,to infer the relation of formula between pressure and location about the squeezed equipment of sugar mud , combining with the theory of compression ratio and volume formula, which provides the theoretical support of designing and manufacture for the device of filter pressing.
\end{abstract}

\section{Introduction}

Filtration and separation is an essential technology to the application of modern industryl,the level of its technology affects the production of equipment and production efficiency directly,it is the foundation of protecting the environmental health,improving economic and social benefits.In general,the materials with high moisture such as municipal sludge,sediment,vinasse, are easier to arouse the attention of public for the dehydration requirement,no matter which way to be adopted,the premised condition is dehydration ${ }^{[1]}$, after that, the area will be covered by less materials and easy to be transported.

The industry of sugar has been widely spread,but its inefficient leads to a trend with highly using of various products in the process of refining sugar to improve the utilization. In the process of refine sugar several kinds of by-product can supply the crops with nutrition in factory,among which filter mud is the most with high moisture,mingles different kinds of coarse protein and cellulose, it's a product that causes bacteria rapidly. The fluid not only contaminates the soil,but permeates into the underground to pollute the water permanently by means of landfill ${ }^{[2]}$. The comprehensive utilization of products and byproducts can bring about more economic benefits rather than waste reduced .The corresponding quality research of filter mud provides a practical reference for design and development of squeezer.

\section{The Experimental Design of Squeeze Filter Mud}

The Experimental Device and Material.As the experimental device shown in Fig.1, it consists of the main body,the cylinder, the piston, the filter cloth and the pressure device.The diameter of cylinder is $100 \mathrm{~mm}$, the height is $250 \mathrm{~mm}$,numerous pores are drilled at the bottom of the main body with $1 \mathrm{~mm}$ to let the fluid flows fluently in the process of experiment.This device is used to make an experiment of filtration under the constant and variable pressure. Within that time,the lever will rotate and form an angle, according to the formula of pressure, the pressure on filter mud remains constant,so it is not necessary to take the angle into consideration.At last, the experimental equipment includes the stopwatch, the electronic scale,the funnel,the sandbags, the weight balance, the measuring cylinder and so on.The moisture of filter mud in experiment is $20.87 \%$ provided by sugar factory. 


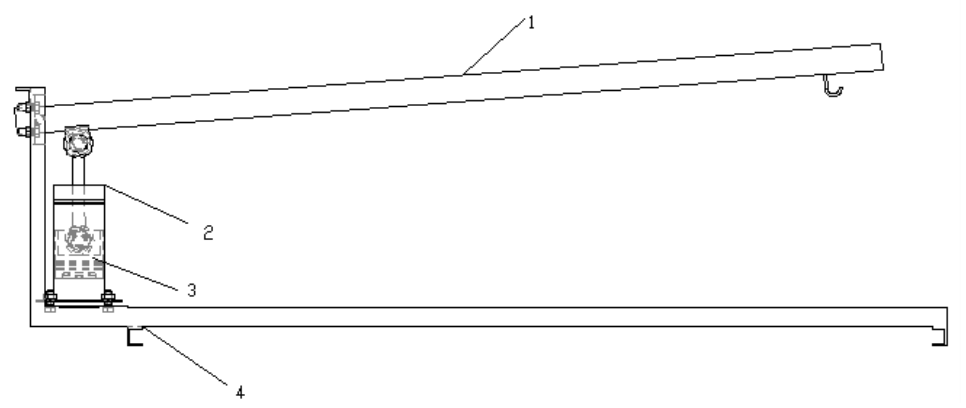

Fig. 1 Schematic figure of device

1-pressure unit 2-cylinder 3-piston 4-main part

Filter Sediment under Constant Pressure.In order to study the relation between volume and the time of sediment for this condition of experiment,eight groups of samples and 700 gram samples of filter mud are put into cylinder respectively,squeezing the mud by loading device and collect the filtrate by funnel and cylinder. And the time is recorded in a certain volume to get the changes in relation of time and volume with different filter cloth in the same pressure.

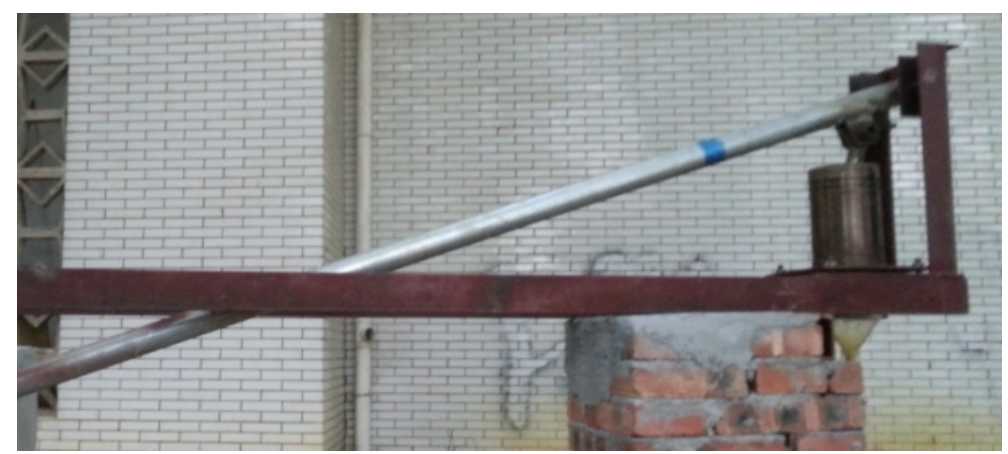

Fig.2 The image of pressure device on the spot

Experimental Results and Analysis. Take the average value respectively in both two experiments of each filter mud samples. The relation between volume and the change of time under the constant pressure is shown in Fig.3.

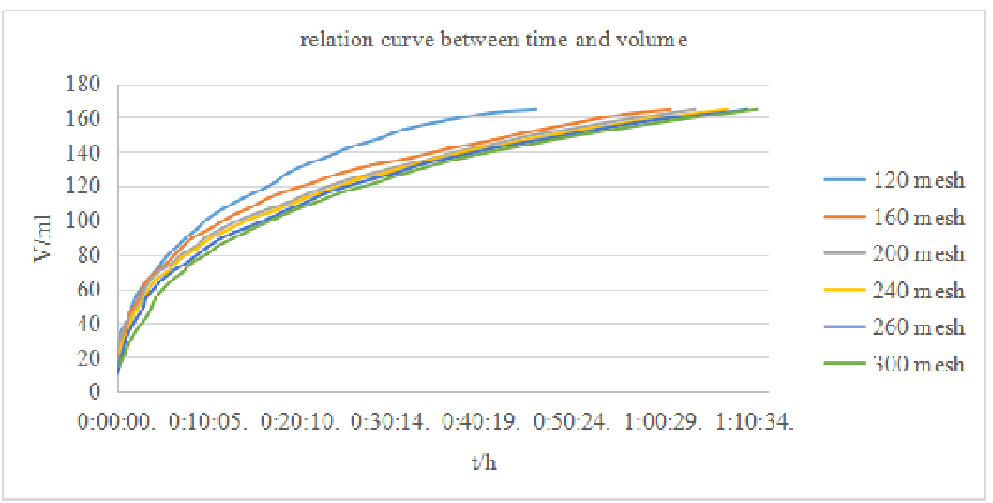

Fig. 3 Curve: relation between time and volume

What can be seen in the Fig. 3 is the condition of the same pressure with $0.56 \mathrm{Mpa}$, the velocity of dewatering is different with various mesh filter cloth,but close to the stable finally.For the less mesh of filter cloth in a certain time,more water collected in the measuring cups, because the filter mud near the filter cloth is more easier to develop into the filter cake in the process of experiment and the distance among the particles becomes short leading to the fluid can not permeate successfully. The result of filter cakes can be seen in the Fig.4.On the contrary, the effect of dehydration is better.In 
conclusion,comparing with the concentration (Fig.5) of fluid,it is obvious to take the next experiment with 260 mesh filter cloth.

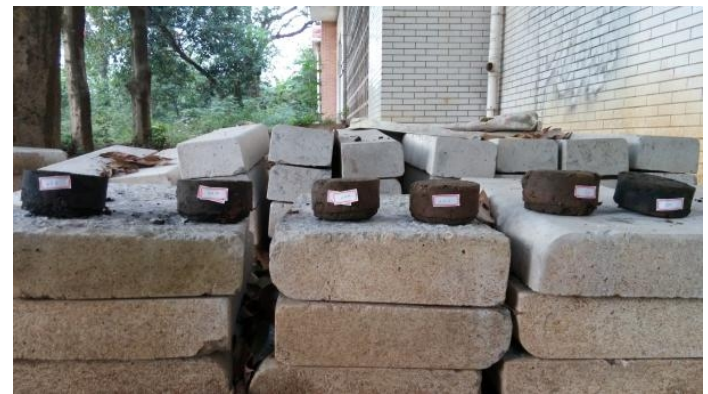

Fig. 4 Comparative map of filter cake

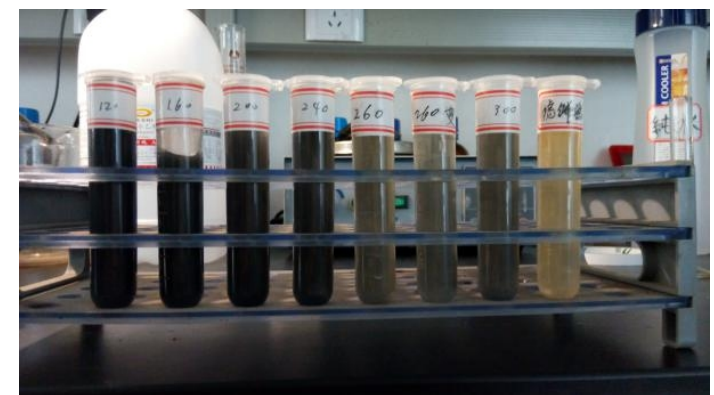

Fig. 5 Comparative map of filter liquor

Filter Sediment under Variable Pressure.In order to study the relation between volume and changed time of sediment for this condition of experiment,taking two groups of sample with 700 gram from filter mud into cylinder respectively,squeezing the mud with all kinds of pressure from $0.21 \mathrm{Mpa}$ to $0.81 \mathrm{Mpa}(0.21 \mathrm{Mpa} \backslash 0.36 \mathrm{Mpa} \backslash 0.51 \mathrm{Mpa} \backslash 0.66 \mathrm{Mpa} \backslash 0.81 \mathrm{Mpa})$, and the time is recorded in a certain volume $(0 \mathrm{ml} \backslash 95 \mathrm{ml} \backslash 125 \mathrm{ml} \backslash 145 \mathrm{ml} \backslash 160 \mathrm{ml})$ to get a graph about relation between time and volume.

Experimental Results and Analysis.Take the average value respectively in both two experiments of each filter mud samples. The relationship between volume and the change of time under the variable pressure is shown in Fig.6.

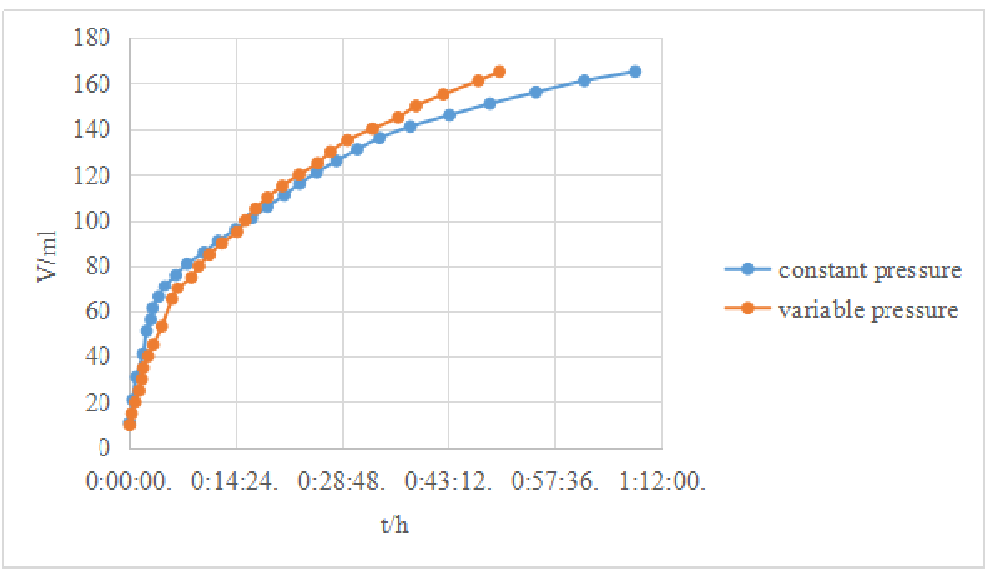

Fig. 6 Curve: relation between time and volume

As shown in Fig 5, at the beginning of the squeeze,the constant pressure is greater than the variable pressure, which means the fast speed of effluent.But after 15 minutes, the total volume with variable pressure overpasses the constant pressure slowly,the distance among the particles becomes short resulting from the constant over-pressure leading to the fluid can not permeate through mud successfully,then the speed slows down.However, under the variable pressure, it's hard to come into a filter cake.As the pressure improving,the speed of fluid squeezed is faster,consuming less time to collect the same volume.

According to the calculation formula of moisture,the initial moisture of filter mud is about $20.87 \%$ through the experiment measure, after a series of squeeze with variable pressure, the moisture reduced to $18.09 \%$. The concentration of fluid with 260 mesh filter cloth is $10.025 \mathrm{~g} / \mathrm{L}$, which conforms to the standard. 


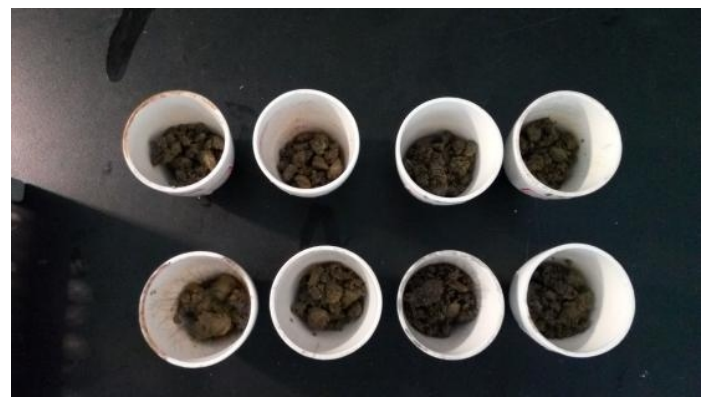

Fig.7 Drying filter mud

Filtration Velocity.Filtration rate is an important index in the process of filter pressing, its numerical value increases with the pressure improving, the changed of filtration rate means filtration resistance and porosity of cake altered ${ }^{[3]}$.In Fig.8, filter mud be squeezed with the constant and variable pressure to get a figure about speed and time,recording the volume by measuring cylinder and corresponding time by stopwatch respectively,analyzing and clearing up the data to get a figure of $v$-t.

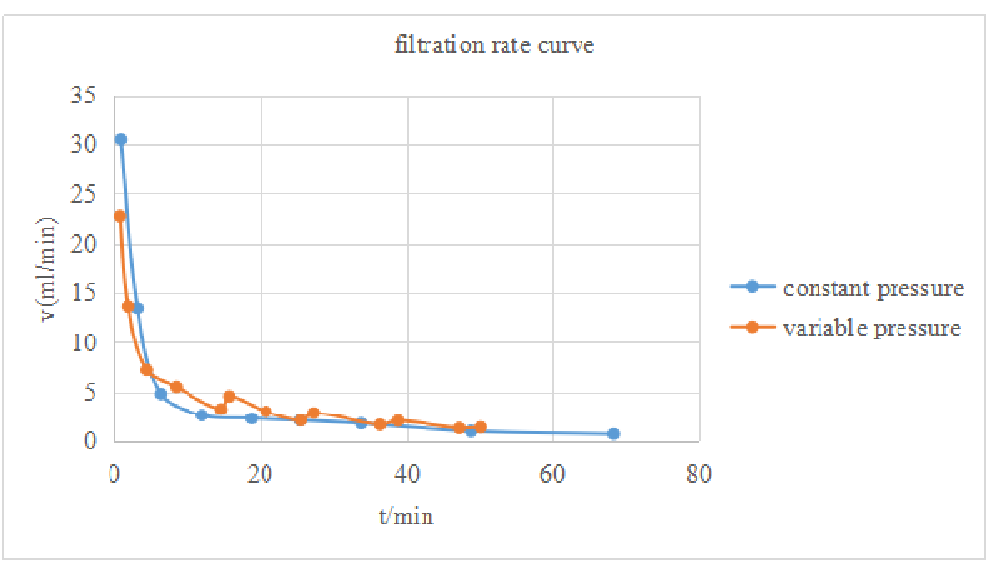

Fig. 8 Curve: filtration rate

From Velocity time curve,regardless of the constant pressure or the variable pressure,filtration rate increases drastically with the time going under the same experimental condition. At the beginning of the process ,the speed decreases from high to low before coming into filter cake, and tends to be horizontal.It can be seen that the rate keeps at a low level from the curve with variable pressure,but it shorten the time on the whole, when the experiment increases the filter pressure, the rate will increase slightly, which means the pressure is a key factor for the filtration rate.

Compression Ratio to Confirm. The compression ratio refers to the ratio of the volume of material before squeezed and of filter mud squeezed, it means a formula, which is a key parameter that represents the performance of filtering equipment,its numerical value not only affect the design of equipment structure,but actual effect of squeeze ${ }^{[4]}$. According to the experimental data,the practical compression ratio is the volume ratio of each stage ,therefore the practical compression ratio of each stage to the first stage is shown in Table 1.

Table 1 The relation between series and actual compression ratio

\begin{tabular}{|c|c|c|c|c|c|}
\hline series & 1 & 2 & 3 & 4 & 5 \\
\hline actual compression ratio & 1.01 & 1.06 & 1.12 & 1.19 & 1.46 \\
\hline
\end{tabular}

So far,according to the experience of practice, the theory of compression ratio is 1.5-4.5 times of practical compression ratio ${ }^{[5]}$. 


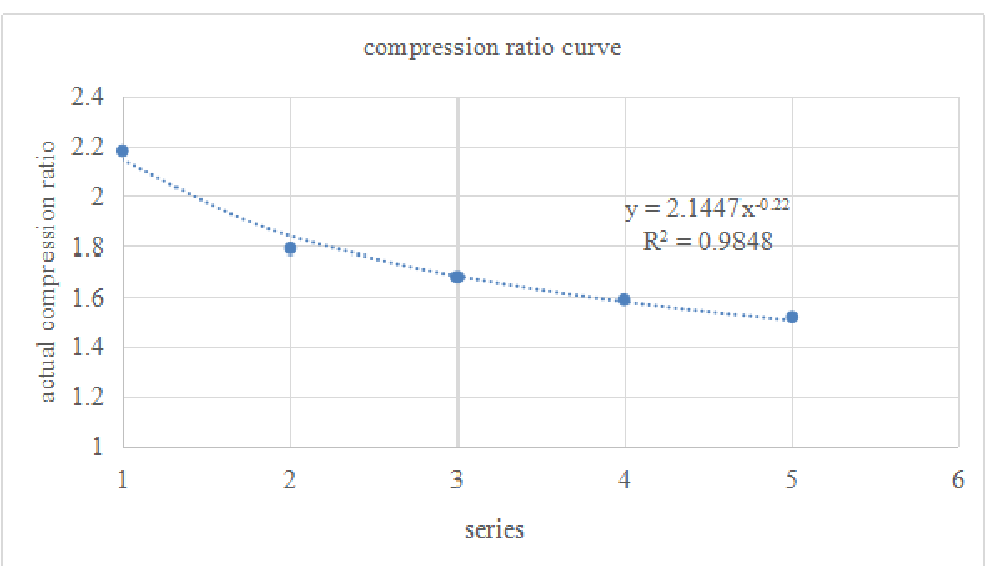

Fig. 9 Curve:compression ratio

According to the fitting curve above,the researches do suggest that the experimental data of compression ratio probably follows a power function

$$
y=2.1447 x^{-0.22} .
$$

\section{Volume Calculation}

The structure of screw press device mainly consists of spiral blade and spindle, the study decides to adopt the screw axis with equal diameters and variable pitches to be convenient of maintenance and save materials ${ }^{[6]}$.The structure has been shown in Fig.10.

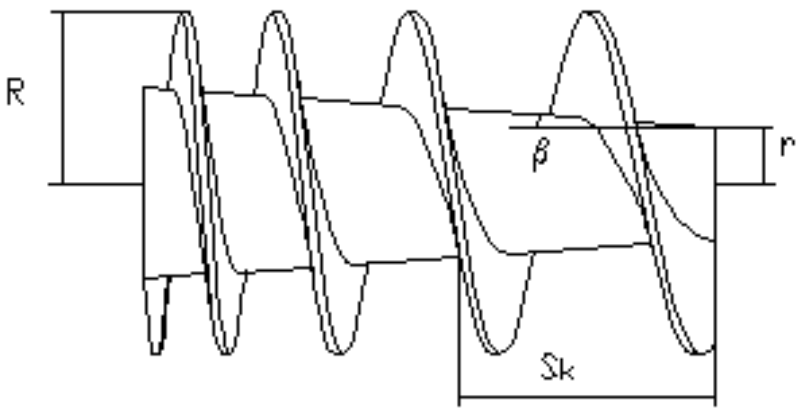

Fig. 10 Schematic figure of spiral structure

For the Kth pitch,the volume can be calculated by the difference between the total volume of pressing cage and the volume of the screw spindle, represented by a mathematical formula

$$
V=\pi\left(S_{\mathrm{k}}-S_{\mathrm{k}-1}\right)\left(R^{2}-\frac{1}{3}\left(\left(r_{k-1}+a\right)^{2}+r_{k-1}^{2}+b\right)\right) .
$$

where

$$
a=\left(S_{\mathrm{k}}-S_{\mathrm{k}-1}\right) \tan \beta, b=r_{k-1}+\left(S_{\mathrm{k}}-S_{\mathrm{k}-1}\right) \tan \beta .
$$

From what has been discussed above, it is obvious to deduce the equation of relation between pressure and location

$$
P=\frac{K \cdot V_{2} / V_{1}}{e^{\eta}}
$$


Where

$$
\begin{aligned}
& \eta=e^{3.4682-\frac{\ln \left(V_{2} / V_{1}\right)}{0.22}} V_{2}>V_{1} \\
& V: \text { volume of the difference between pressing cage and screw spindle. }
\end{aligned}
$$

The formula of pressure and compression ratio that can be expressed by equation

$$
P=k \cdot \varepsilon / e^{n},
$$

combining with the formula of volume.

\section{Conclusions}

In order to understand the basic dehydration qualities about filter mud,the study analysis the relation of curve between volume and time under the constant pressure by sediment squeezed.Based on fluid concentration, choosing filter cloth with 260 mesh,it can achieve a satisfactory performance.

In view of the experiment about squeeze under variable pressure, if the study adds too much pressure to the filter mud instantaneously, which is easy to form filter cake,result in liquid flowing slowly.The study adopts squeeze with variable pressure for filter mud,increasing the pressure in the process of experiment which prompts the filtration rate and the dehydration rate is most obvious, its moisture reduced to $18.09 \%$.

According to the volume changes about filter mud in the process of experiment with variable pressure,combining with the theory of compression ratio to deduce the equation of relation between pressure and location on the structure of spiral.

The study fully understands the dehydration qualities about filter mud on various aspects by the experiment,which provides a certain theoretical basis for theory research and equipment development,has a great practical significance.

\section{References}

[1] Hong-bin Yuan.High Moisture Content Biomass Dewatering Technology Research[D].Nanjing University of Science \& Technology.( 2012).

[2] Wei-wei Wang.Investigation on the Mechanical and Thermal Expression Dewatering of Filter Mud and Its Combustional Characteristics [D]. Guangxi University, (2014).

[3] Yang Zhao.Study on the Microstructure of the Filter Cake and the Expression Filtration Theory [D].Zhejiang University, (2006).

[4] Li-yan Yang.the Theoretical Research and Analysis on Ratio of Screw Presser[D].Henan University of Technology,(2013).

[5] Peng-fei Wei,Hai Xiang,Shu-zheng Hu,Ren-sheng Mu,Jia-jia Ren,Yan-tong Yu.Discussion of Key Design of Screw Expeller[J].Journal of Agricultural Mechanization Research,( 2013),11:92-94+98.

[6] Nian- meng Zhang,Qing- wen Qu,Yun- hua Chen.Mathematical Model Research of Screw Axis Applied in Screw Press Based on Dehydration Parameters[J].Coal Technology, (2015), 02:241-243.

[7] Zhi-qing Li.How to Choose the Series of Oil Mill in a Science Way [J].Technological Development of Enterprise,( 2014),27:93-94+106. 